\title{
Morfología espacial y objetual I: Taxonomías espaciales y objetuales en espacios y productos I Proyecto de Investigación \\ $\mathrm{N}^{\circ} 3.2$ \\ Equipo de Investigación ${ }^{(1)}$ \\ Ana Cravino y Roberto Céspedes \\ por Facultad de Diseño y Comunicación, \\ Universidad de Palermo (ARG)
}

\begin{abstract}
Resumen: El Proyecto de Investigación 3.2 presenta e invita a una reflexión acerca de aquellos momentos en los que la sociedad genera cambios significativos en distintas disciplinas buscando un discurso en común. Reúne dos aproximaciones interesantes sobre temas fuertemente vinculados con el diseño en ese momento histórico en el que se producen modificaciones centrales en la lógica proyectual: el comienzo de la modernidad. Los últimos años del siglo XIX y los primeros del XX representaron una de las épocas más innovadoras en el diseño y la arquitectura. Acompaña los cambios culturales igualmente trascendentes y disruptivos que introdujeron al mundo en la Modernidad. Esta modificación de los principios rectores del diseño representó un punto de inflexión insoslayable y al que, por continuidad o quiebre, nos seguimos refiriendo hasta el día de hoy.
\end{abstract}

Palabras clave: Morfología - Espacio - Objeto - Modernidad - Racionalismo - Función Expresión - Historia del diseño.

[Resúmenes en inglés y portugués en la página 84]

${ }^{(1)}$ Los CVs del Equipo de Investigación pueden consultarse en el Capítulo Directores de Líneas y Coordinadores de Proyectos de esta misma Edición.

\section{Acerca del Proyecto 3.2}

Morfología espacial y objetual I

Taxonomías espaciales y objetuales en espacios y productos

El Proyecto 3.2 Morfología espacial y objetual I: Taxonomías espaciales y objetuales en espacios y productos, reúne diversas perspectivas donde los investigadores, provenientes de distintas disciplinas, como arte, arquitectura o diseño en general, plantean categorizar 
las particularidades de la forma, tanto desde fuera en los objetos como en los espacios y contrastarlas con producciones de los últimos tiempos para analizar posteriormente los puntos de contacto. El Proyecto 3.2 proviene de la reorientación de una investigación perteneciente al Ciclo A (2011 - 2015) denominada Diseño de Interiores en la Historia, que finalizada, el equipo de investigadores se propuso continuar con la exploración centrada en el estudio de la forma, con el fin de impactar en la actualización de contenidos de los programas académicos de las carreras de grado relacionados con el diseño de productos y espacios y orientar la formación profesional hacia una mayor significación de la forma y la materialización de los diseños. Esta propuesta aborda diversas miradas sobre las incidencias de los nuevos avances tecnológicos sobre materiales y procesos en disciplinas como Diseño de Interiores, Industrial, de Indumentaria y Arquitectura.

Las reflexiones y los resultados obtenidos en el Proyecto 3.2 Morfología espacial y objetual I, se continúan desarrollando en los Proyectos 3.4 Morfología espacial y objetual II, 3.6 Morfología espacial y objetual III y el 3.7 Creatividad, Emoción y Espacio, asimismo guarda relación y comparte alguno de sus investigadores con los Proyectos 3.1 Materialidad Difusa, 3.3: Identidad Difusa y 3.5 Profesionalización Difusa en el marco de la misma Línea de Investigación y bajo la misma Directora Ana Cravino.

Sus principales objetivos son:

- Reflexionar sobre los instrumentos conceptuales que permiten discernir e intencionarla producción de significados intrínsecos al diseñoyla materialidad delos objetos. - Explorar distintas perspectivas sobre la incidencias de los nuevos avances tecnológicos sobre materiales y procesos en disciplinas cómo Arquitectura, Diseño de Interiores, de indumentaria e Industrial.

\section{Acerca de la Línea 3}

Forma y Materialidad: Incidencia de la morfología y la tecnología en el diseño de espacios y productos, dirigida por Ana Cravino se desarrolla de manera ininterrumpida desde 2015 en la Facultad de Diseño y Comunicación (UP, Argentina), e incluye hasta el momento siete proyectos finalizados, el 3.1 Materialidad Difusa y 3.3 Identidad Difusa coordinados por Daniel Wolf (UP) y Luisa Collina (Instituto Politécnico de Milán, Italia); el 3.5 Profesionalización Difusa coordinado por Fabiola Knop y Alejo García de la Cárcova (UP) junto a la Universidad Nacional de Mar del Plata (Argentina) y la Universidad Rafael Landivar (Guatemala); el 3.2 Morfología espacial y objetual I coordinado por Ana Cravino y Roberto Céspedes (UP); el 3.4 Morfología espacial y objetual II coordinado por Ana Cravino y Roberto Céspedes (UP), Taña Escobar Guanoluisa (Universidad Técnica de Ambato, Ecuador) y César Giovanny Delgado Banegas (Universidad del Azuay, Ecuador); el 3.6 Morfología espacial y objetual III coordinado por Ana Cravino (UP) y Genoveva Malo (Universidad del Azuay, Ecuador); y el 3.7 Creatividad, Emoción y Espacio coordinado por Sandra Navarrete (Universidad de Mendoza, Argentina). 


\section{Mapa de Áreas y Proyectos}

El Proyecto 3.2 se vincula con todas las carreras de grado y posgrado correspondientes a la Facultad de Diseño y Comunicación, y específicamente Diseño de Interiores, Diseño de Moda y Diseño Industrial de la propia unidad académica y la carrera de Arquitectura de la Facultad del mismo nombre.

\section{Productos y Resultados}

\section{a)- Publicaciones}

Cuadernos del Centro de Estudios de Diseño y Comunicación No81. (2020) Taxonomías espaciales y objetuales en espacios y productos Tesis de Doctorado en Diseño UP recomendada para su publicación. Coordinación Ana Cravino. Año XXI, Septiembre 2020, Buenos Aires, Argentina. ISSN: 1668-0227. Esta publicación documenta y comunica los resultados alcanzados en el Proyecto 3.2 Morfología espacial y objetual. Taxonomías espaciales y objetuales en espacios y productos, y a continuación se detallan los autores y artículos contenidos en ella:

Céspedes, Roberto (2020) Introducción (Pp. 11-13)

Cravino, Ana (2020) Prólogo (Pp. 15-17)

Pokropek, Jorge (2020) Lógicas de coherencia para la interpretación y producción del diseño interior y sus criterios de selección de formas objetuales (Pp. 19-29)

Cravino, Ana La Bolsa de Comercio de Buenos Aires. Un caso paradigmático de composición clásica (Pp. 31-48)

Céspedes, Roberto (2020) Diseño Andrógino: Charles Rennie Mackintosh (Pp. 49-60)

Woodhull, Claudia Marcela (2020) Una aproximación morfológica: formas de la pradera y su intencionalidad estética en el espacio interior y el objeto (Pp.61-78)

Viveros Baez, Ricardo José (2020) Organicismo: morfología y materialidad como expresión comunicante en un espacio arquitectónico (Página 79-97)

Compte Guerrero, Florencio (2020) Tesis recomendada para su publicación. Modernos sin modernidad. Arquitectura de Guayaquil 1930-1948 (Pp. 99-276)

Actas de Diseño No24. (2018) Publicación de las ponencias de la Comisión Forma y Materialidad. En: XII Encuentro Latinoamericano de Diseño "Diseño en Palermo". Foro de Escuelas de Diseño - XI Plenario 2017, VIII Congreso Latinoamericano de Enseñanza del Diseño. II Coloquio de Investigación y Desarrollo en Diseño Latino. Coloquio de Investigación. Julio 2017, Buenos Aires, Argentina. En esta publicación se documentan las ponencias presentadas en la Comisión de la Línea de Investigación No3 Forma y materialidad: Incidencia de la morfología y la tecnología en el diseño de espacios y productos en el II Coloquio de Investigación y Desarrollo en Diseño (Pp. 43-44). 
Escritos en la Facultad No122. (2016) Articulación entre Investigación y Posgrados, Edición 1. Doctorado en Diseño. Maestría en Gestión del Diseño. Programa de Investigación y Desarrollo en Diseño Latino. Línea de Investigación N³: Forma y Materialidad. Incidencia de la morfología y la tecnología en el diseño de espacios y productos. ISSN: 1669-2306. ISSN (En línea): 2591-3743. En esta publicación la Directora Ana Cravino presenta la línea de investigación No3 Forma y materialidad: Incidencia de la morfología y la tecnología en el diseño de espacios y productos, y anuncian el comienzo de los Proyectos 3.1 Materialidad Difusa: Prácticas de Diseño y Tendencias y 3.2 Morfología espacial y objetual. Taxonomías espaciales y objetuales en espacios y productos (Pp. 10).

\section{b)- Congresos / Coloquios / Plenarios}

III Coloquio de Investigación y Desarrollo en Diseño Latino. Universidad de Palermo, 30 de julio de 2018. IX Congreso Latinoamericano de Enseñanza del Diseño. Semana Internacional del Diseño en Palermo.

En la comisión Forma y materialidad, se presentaron las reflexiones y resultados del proyecto 3.2 Morfología espacial y objetual. Taxonomías espaciales y objetuales en espacios y productos, expusieron: Jorge Pokropek, Ricardo José Viveros Báez, Claudia Marcela Woodhull, Roberto Céspedes, Ana Cravino

A continuación se detallan las ponencias presentadas en esta Comisión coordinada por la Directora del proyecto Ana Cravino:

Céspedes, Roberto

Diseño andrógino: Charles Rennie Mackintosh

Cravino, Ana

La Bolsa de Comercio de Buenos Aires. Un caso paradigmático de composición clásica

Pokropek, Jorge

Lógicas de coherencia para la interpretación y producción del diseño interior y sus criterios de selección de formas objetuales.

Viveros Báez, Ricardo José

Organicismo, morfología y materialidad como expresión comunicante en un espacio arquitectónico.

Woodhull, Claudia Marcela

Una aproximación morfológica: Formas de la Pradera y su Intencionalidad Estética en el Espacio Interior y el Objeto

$1^{\circ}$ Plenario de Directores de Investigación DC, 13 de octubre - 2017. Es este primer plenario los Directores de Líneas y Proyectos de Investigación presentaron a sus pares y al conjunto del Programa de Investigación de la Facultad de Diseño y Comunicación, los resultados obtenidos y/o en proceso (publicaciones y acuerdos), junto con los Proyectos. La Directora de la Línea de Investigación No3, presentó a sus pares, los principales avances del Proyecto 3.1 Materialidad Difusa y 3.2 Morfología espacial y objetual. 
II Coloquio de Investigación y Desarrollo en Diseño Latino. Universidad de Palermo, 31 de julio de 2017. VIII Congreso Latinoamericano de Enseñanza del Diseño. Semana Internacional del Diseño en Palermo.

En la comisión Forma y materialidad, se presentaron los avances del Proyecto 3.2 Morfología espacial y objetual, y las reflexiones y resultados del 3.1 Materialidad Difusa, expusieron: Ana Cravino, Roberto Céspedes, Daniela Di Bella, Clelia Domoñi, Martin Isidoro, Omar Montero, Jorge Pokropek, Ricardo José Viveros Báez

A continuación se detallan las ponencias presentadas en esta Comisión coordinada por la Directora de la línea Ana Cravino:

Cravino, Ana

Morfología espacial y objetual

Céspedes, Roberto

El modernismo japonés en la Hill house de Helenburgh y la silla Mackintosh de Charles Mackintosh

Di Bella, Daniela

Lectura del orden y el desorden

Domoñi, Clélia

Reconstruir el espacio del edificio Crystlals de las Vegas y el producto en la lámpara Paragon del Arq. Daniel Libeskind

Isidoro, Martín

El neoplasticismo de la casa Schröder en Utrecht y la silla roja y azul de Gerrit Rietveld.

Montero, Omar

Morfología espacial y objetual

Pokropek, Jorge

Taxonomía de la forma y el espacio

Viveros Báez, Ricardo José

El organicismo de Alvar Aalto

I Coloquio de Investigación y Desarrollo en Diseño Latino. Universidad de Palermo, 26 de Octubre de 2016.

Se presentó la Línea de Investigación No3: Forma y Materialidad, Incidencia de la morfología y la tecnología en el diseño de espacios y productos, como una de las líneas que integran el Programa de Investigación en Diseño de la Facultad de Diseño y Comunicación.

Cravino, Ana (Argentina) Presentación de la Línea de Investigación No3: Forma y Materialidad. Facultad de Diseño y Comunicación, Universidad de Palermo.

\section{c)- Formación de Posgrado e Impacto curricular}

La Directora Ana Cravino es Docente de Posgrado en Diseño de la Facultad de Diseño y Comunicación en Maestría en Gestión del Diseño y Doctorado en Diseño y al igual que Roberto Céspedes (UP) incorporan los contenidos de su investigación a sus asignaturas en posgrado. 


\title{
Evaluación Externa
}

El Proyecto 3.2 Morfología espacial y objetual I: Taxonomías espaciales y objetuales en espacios y productos, cuyos resultados fueron publicados en el Cuaderno No81, fue evaluado exitosamente por la Evaluadora María del Rosario Bernatene en 2019.

\begin{abstract}
Research Project 3.2 presents and invites a reflection on those moments in which society generates significant changes in different disciplines, seeking a common discourse. It brings together two interesting approaches on themes strongly linked to design at that historical moment in which central modifications in the design logic take place: the beginning of modernity. The last years of the 19th century and the first of the 20th represented one of the most innovative times in design and architecture. It accompanies the equally transcendent and disruptive cultural changes that introduced the world in Modernity. This modification of the guiding principles of design represented an unavoidable turning point and to which, due to continuity or break, we continue to refer to today.
\end{abstract}

Keywords: Morphology - Space - Object - Modernity - Rationalism - Function - Expression - History of design.

Resumo: O Projeto de Pesquisa 3.2 apresenta e convida a uma reflexão sobre aqueles momentos em que a sociedade gera mudanças significativas em diferentes disciplinas, buscando um discurso comum. Reúne duas abordagens interessantes sobre temas fortemente vinculados ao design naquele momento histórico em que ocorrem modificações centrais na lógica do design: o início da modernidade. Os últimos anos do século XIX e os primeiros do século XX representaram um dos momentos mais inovadores do design e da arquitetura. Ele acompanha as mudanças culturais igualmente transcendentes e disruptivas que introduziram o mundo na Modernidade. Esta modificação dos princípios orientadores do design representou um ponto de inflexão inevitável e ao qual, por continuidade ou ruptura, continuamos a nos referir hoje.

Palavras chave: Morfologia - Espaço - Objeto - Modernidade - Racionalismo - Função Expressão - História do design.

[Las traducciones de los resúmenes fueron realizadas a través de traductor automático] 